\title{
Handmade Infrared Rays Complementary Metal Oxide Semiconductor (IR-CMOS) Camera for Recording Eye Movements
}

\author{
Fumiyuki Goto* and Youji Asama
}

Department of Otorhinolaryngology, Hino Municipal Hospital, Tokyo, Japan

\begin{abstract}
Objective: It is important to observe eye movements in order to evaluate peripheral or central vestibular disorders in dizzy patients. An infrared rays charge coupling device (IR-CCD) camera is now widely used in daily practice. The technical advantage of a complementary metal oxide semiconductor (CMOS) now allows us to develop an inexpensive handmade camera for carrying out nystagmus recordings.

Methods: We developed a handmade IR-CMOS camera at a reasonable cost of less than $\$ 150$. The costs of a small IRCMOS camera, scuba diving goggles, an AC adapter, an audiovisual (AV) cord, and a plastic board with a thickness of $0.5 \mathrm{~mm}$ were $\$ 60, \$ 60, \$ 10, \$ 10$, and $\$ 10$, respectively. It takes almost an hour to assemble the camera. We recorded eye movements using this camera.

Results: The image obtained using this inexpensive handmade camera was clear and highly comparable to that obtained using a conventional IR-CCD camera.

Conclusion: The handmade IR-CMOS camera is inexpensive, easy to develop, and can be effectively used in daily practice. In future, with technical developments in electronics, we intend to develop a more compact and high-quality camera for recording eye movements.
\end{abstract}

Keywords: Infrared rays, CMOS, eye movements.

\section{INTRODUCTION}

Conventionally, Frenzel glasses [1] had been used to examine nystagmus and dizzy patients. Recently, an infrared rays charge coupling device (IR-CCD) camera is widely used in daily practice [2-5]. However, since this camera is expensive, not all doctors have it. The technical advantage of a complementary metal oxide semiconductor (CMOS) now allows us to develop an inexpensive handmade camera for carrying out nystagmus recordings.

\section{MATERIAL AND METHODS}

In order to record eye movements, we developed handmade goggles at a reasonable cost of less than $\$ 150$ using all the commercially available materials. These goggles were equipped with a small IR-CMOS camera. This camera was SS2000BLM (Kyoritsu, Osaka, Japan). The specification of this camera is that it has a total pixel of 542 (horizontal) $\times 492$ (vertical), a resolution of $380 \mathrm{TV}$ lines (horizontal), and a weight of $25 \mathrm{~g}$. The scuba diving goggles were Quarts 2 mask (Black/Black) (AQUALUNG Japan, Knagagawa, Japan). Costs of each of the materials that include a small IR-CMOS camera, goggles, an AC adapter, an audiovisual (AV) connection cord, a and plastic board with a thickness of $0.5 \mathrm{~mm}$ were $\$ 60, \$ 60, \$ 10, \$ 10$, and $\$ 10$, respectively. First, bilateral lenses of the goggles were detached. These lenses were replaced with a black plastic

*Address correspondence to this author at the Department of Otorhinolaryngology, Hino Municipal Hospital, 4-3-1 Tamadaira, Hino-city, Tokyo, 191-0062, Japan; Tel: +81-42-581-2677; Fax: +81-42-581-2923;

E-mail: amifumi@bc5.so-net.ne.jp board. The design of the plastic board for the left eye is shown in Fig. (1). Then, the AC adapter was connected to the power supply. The AV output was connected to a video deck and then to a TV monitor using an AV cord.

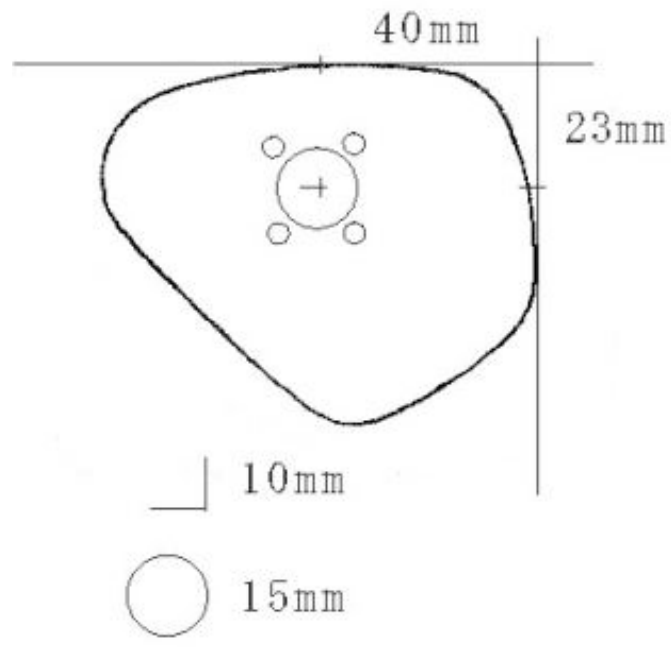

Fig. (1). Design of a lens of the left eye. Design of a lens of the left eye is shown. The holes shown are created using a drill.

\section{RESULTS}

It took less than an hour to assemble the camera. The weight of the camera with the goggles was 200 g. Fig. (2) shows the developed handmade goggles. Fig. (3) shows the result of recording the eye movements using this camera. The obtained image was clear. The quality of the image 
obtained using this camera was highly comparable to that obtained using a conventional IR-CCD camera. This camera also has a small microphone, and it is possible to record voice simultaneously. The weight of the camera with the goggles was only $200 \mathrm{~g}$, which was sufficiently light for daily use.

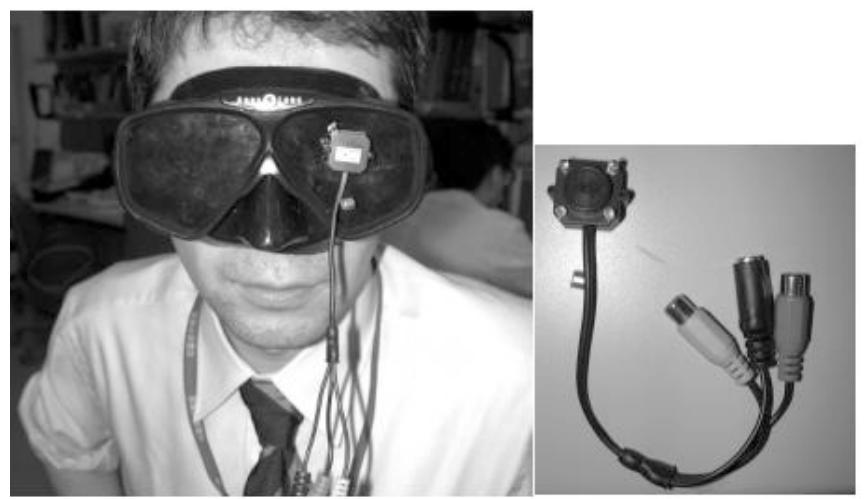

Fig. (2). Photograph of the CMOS camera with the handmade goggles. CMOS camera is securely equipped on the scuba diving goggles.

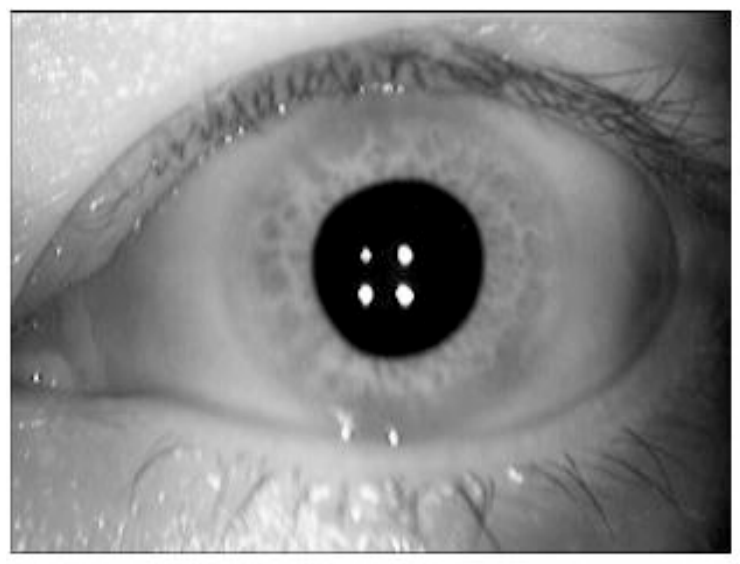

Fig. (3). Result of recording eye movements. Quality of the image obtained was almost same as that obtained using the conventional IR-CCD camera.

\section{DISCUSSION}

The CMOS camera is not only small but also inexpensive with a low electronic consumption. This camera can be easily purchased via the Internet. It is very easy to assemble this camera, and a layman who does not have any knowledge of basic electronics can also assemble it.

\section{CONCLUSIONS}

The CMOS camera is a high-quality camera, and it can be effectively used in daily practice. In future, with technical developments in electronics, we intend to develop a more compact and high-quality camera for recording eye movements.

\section{REFERENCES}

[1] Frenzel, H. Spontan- und Provocations-Nystagmus als Krankheitssymptom: ein Leitfaden fur seine Beobachtung in Aufzeichnung und Formnalyse. Springer: Berlin 1955; pp. 1-72.

[2] Funabiki, K., et al. Light-weight and low-cost infrared CCD eye monitoring system designed for routine vestibular clinic use. Acta Otolaryngol Suppl 1997; 528: 67-9.

[3] Nagao, S. A non-invasive method for real-time eye position recording with an infrared TV-camera. Neurosci Res 1990; 8(3): 210-3.

[4] Vicini C, Campanini A, Cuiffolotti R, Armato E, Ferri E, Ulmer E. Innovations, advantages and limitations of the infrared videonystagmography. Acta Otolaryngol Ital 2003; 23(6 Suppl 76): 3-7.

[5] Yamanobe, S, Taira S, Morizono T, Yagi T, Kmio T. Eye movement analysis system using computerized image recognition. Arch Otolaryngol Head Neck Surg, 1990. 116(3): 338-41.

This is an open access article licensed under the terms of the Creative Commons Attribution Non-Commercial License (http://creativecommons.org/licenses/by$\mathrm{nc} / 3.0 /$ ) which permits unrestricted, non-commercial use, distribution and reproduction in any medium, provided the work is properly cited. 